\title{
In the eye of the beholder
}

\section{Scientific journals and regulatory agencies want reliable clinical trials, but the definition of 'reliable' isn't necessarily the same for both parties.}

L

ast month, during a translational-research conference, a researcher complained that the New England Journal of Medicine had just rejected his latest clinical trial. Apparently, the journal thought that although the results were encouraging, the number of patients enrolled in the trial was too low to draw definitive conclusions from the study.

The scientist was not complaining that the journal had been unfair with his work, and agreed that the trial had fewer than optimal patients. The problem was that when he presented his investigational new drug (IND) application to the US Food and Drug Administration (FDA), safety concerns forced him to change the trial design and reduce the number of subjects to receive approval.

Both the journal and the FDA have good reasons to emphasize different aspects of the trial—scientific accuracy and patient protection. Unfortunately for researchers, these divergent opinions can place them between a rock and a hard place. Even more unfortunate, discrepancies of this sort are not all that infrequent.

According to the FDA, phase 1 trials "are designed to determine the metabolism and pharmacologic actions of the drug in humans, the side effects associated with increasing doses, and, if possible, to gain early evidence on effectiveness." An exploratory clinical trial, in turn, can also provide pharmacological information but "is conducted early in phase 1 , involves very limited human exposure, and has no therapeutic or diagnostic intent."

At Nature Medicine, we receive many papers reporting exploratory trials. And the FDA definition notwithstanding, the primary endpoint of most of them is efficacy. Dosing and metabolism are often secondary endpoints and occasionally not even part of the submission. Nevertheless, we would be very reluctant to publish an exploratory trial that did not include efficacy data, and this is also true for other journals that publish translational studies.

Another example of differences between the criteria of journals and the position of regulatory agencies comes from Europe, where ethical concerns prevent the approval of placebo-controlled trials if there is another drug that can act as 'active comparator'. Many reviewers, however, are not persuaded by this explanation and advise the journal against publishing the trial.

It would be unfair to say that regulatory agencies have not tried to be more supportive of clinical researchers. For example, the FDA has mechanisms to deal with clinical trials that use active comparators, acknowledging the ethical problems associated with some placebocontrolled trials. Also, last January, the FDA issued the 'Guidance for Industry, Investigators and Reviewers of Exploratory IND Studies'. This document highlighted the great deal of flexibility that existing regulations allow in the amount of data needed for the approval of an IND application, depending on the goals of the research, the specific testing proposed and the expected risks.

But despite this welcome flexibility, the emphasis is still on safety, as the document largely referred to microdosing studies designed to confirm that the expected mechanism of a drug's action can also be observed in humans, to measure binding affinity or localization of the compound, or to assess pharmacokinetics of the drug.

It would be absurd to argue that the preoccupation of regulatory agencies with patient welfare is unwarranted. The harsh criticisms that the FDA has experienced after reports of serious side effects of approved drugs are stark reminders of the need to constantly monitor safety. But similarly, it is difficult to argue that highly visible journals should lower their standards when evaluating clinical trials. Publishing an exploratory trial can have a profound effect on subsequent clinical testing, the same way that a large-scale trial can profoundly influence drug approval and clinical practice. Using less rigorous publication standards cannot be beneficial for the scientific and clinical communities, and ultimately for the public at large.

At Nature Medicine we have tried to raise our standards for exploratory trials, emphasizing the need for a control arm, encouraging the use of a blinded testing design, and asking for the inclusion of as many patients as possible. We appreciate that meeting these conditions is not always possible (particularly for very rare diseases), and can therefore be occasionally flexible. But in general terms, we turn down most of the studies that do not meet our criteria.

It is somewhat paradoxical that despite the current research climate of frank support of bench-to-bedside studies, scientists find it hard to get their clinical trials approved. And, when they get them approved and finally complete them, it becomes problematic to publish them in high-profile journals. Alas, there does not seem to be an easy way out of this conundrum. One thing is quite clear, though: this roadblock to translational research does not bode well for the development of new medicines. 